\title{
Intangibles Kapital und Rentabilität in der Pharmaindustrie
}

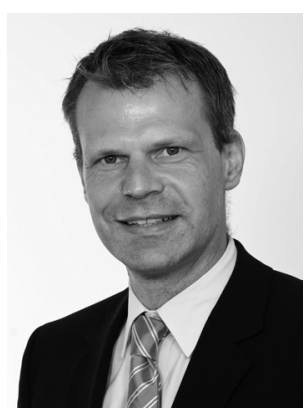

\section{Jörg C. Mablich und Burcin Yurtoglu}

F\&E-Investitionen, Rentabilität, intangibles Kapital, Pharmaindustrie R\&D investments, profitability, intangible capital, pharmaceutical industry

Die Pharmaindustrie gehört auf den ersten Blick zu den rentabelsten Branchen weltweit. In unserer Studie gehen wir den Ursachen der hohen Rentabilität nach. Während in älteren Erklärungsansätzen Marktmacht und Markteintrittsbarrieren eine grosse Rolle spielten, führen neuere Arbeiten hohe Rentabilität in forschungsintensiven Branchen auf Messschwierigkeiten zurück, die dadurch entstehen, dass Forschungs- und Entwicklungsinvestitionen als Aufwand anstatt als Vermögensgegenstand mit anschliessenden Abschreibungen behandelt werden. Unter Verwendung von internationalen Firmendaten aus den Jahren 1985 - 2004 aktivieren wir in unserer Arbeit die Forschungs- und Entwicklungsinvestitionen und berechnen die korrigierte Rentabilität auf den um Forschungs- und Entwicklungsinvestitionen ergänzten Kapitalstock. Die Rentabilität von Pharmafirmen sinkt dabei um zwei bis fünf Prozentpunkte und liegt somit auf ähnlichem Niveau wie die von anderen forschungsintensiven Branchen.

In this paper we try to explain the high profitability of the pharmaceutical industry. While older explanations emphasize the importance of market-entry barriers and monopoly power, we argue that it is mainly due to measurement errors that arise from the treatment of RహD investments and intangible capital by conventional accounting methods. In an empirical exercise we show that profit rates drop by two to five percentage points when capital stocks are calculated to include intangible R\&D capital.

\section{Problemstellung}

Der Pharmaindustrie wird in der gesundheitspolitischen Debatte oftmals eine hohe Rentabilität aufgrund festgelegter Arzneimittelpreise und somit auf Kosten der Versichertengemeinschaft unterstellt (Schäppi 2002; Angell 2004). Marcia Angell, emeritierte Medizinprofessorin der Harvard-Universität und ehemalige Chefredakteurin des angesehenen New England Journal of Medicine spricht in einem Interview mit dem Schweizer Magazin Die Weltwoche kritisch von den „rekordverdächtigen Gewinnen“ der Pharmaindustrie (Die Weltwoche 2004). Auf den ersten Blick zeichnet sich die Pharmaindustrie tatsächlich durch eine gute Gewinnsituation im Vergleich zu anderen Branchen aus. So glänzt in der Fortune-500 Liste des Jahres 2001 die Pharmaindustrie mit einer Rentabilität auf die Bilanz- 
summe von 17,7 Prozent, während der Medianwert für alle 500 untersuchten Unternehmen bei lediglich 3,9 Prozent lag. Auch im Zeitraum 2006- 2009 lag die Pharmaindustrie in der Fortune-Liste von 52 Branchen immer unter den besten fünf. Im Krisenjahr 2009 war die Rendite der Pharmaindustrie sogar knapp sechs Mal höher als der gesamtwirtschaftliche Median (Fortune 2010). Wissenschaftliche Untersuchungen kommen zu ähnlichen Ergebnissen und attestieren der Pharmaindustrie eine dauerhafte, signifikant über dem Durchschnitt liegende Rentabilität (Mueller 1986; Odagiri/Yamawaki 1990; Gschwandtner/Cable 2008). Wie lässt sich die beobachtete hohe Rentabilität ökonomisch erklären? Es werden in der Literatur drei wesentliche Erklärungsansätze angeführt.

1. Marktmacht: In auf Bain (1951, 1956) zurückgehenden industrieökonomischen Arbeiten werden die überdurchschnittlichen Rentabilitäten mit Marktmacht erklärt, die sich etwa durch Marktkonzentration und Markteintrittsbarrieren ergeben (Comanor/Wilson 1967; Porter 1974). Die Hypothese ist, dass eine hohe Marktkonzentration im Zusammenspiel mit Markteintrittsbarrieren - Bain (1956) nennt hier Kostenvorteile, Grössenvorteile und Produktdifferenzierungsvorteile - Preisabsprachen und Kollusion erleichtern und daher Unternehmen in stark konzentrierten Märkten eine höhere Rentabilität aufweisen. Empirische Arbeiten finden jedoch nur einen kleinen Effekt der Marktkonzentration auf die Rentabilität, der im Zeitverlauf sogar noch abgenommen haben dürfte (Schmalensee 1989; Domowitz et al. 1986). In Deutschland und vielen anderen Ländern gilt daher auch das „weite Oligopol“ als wettbewerbspolitisches Leitbild, und Monopolgewinne dürften nur in wenigen Branchen - meist handelt es sich um die ehemals staatlichen Netzwerkindustrien wie Energieversorgung oder Telekom - eine Rolle gespielt haben.

2. Risiko: Eine weitere Erklärungsmöglichkeit für die hohe Rentabilität der Pharmaunternehmen ist das mit pharmazeutischen Forschungs- und Entwicklungsinvestitionen (F\&EInvestitionen) verbundene hohe Risiko, welches durch eine höhere erwartete Rendite ausgeglichen werden muss. Wie Grabowski/Vernon (1990, 2003) zeigen, erwirtschaftet lediglich ein Drittel aller neu auf den Markt kommenden Medikamente die entsprechenden F\&EInvestitionen. Die F\&E-Investitionen für ein neues Medikament betragen Schätzungen zufolge zwischen 500 Mio. USD und 800 Mio. USD (Adams/Brantner 2006; DiMasi/Hansen/ Grabowski 2003). Von positiven Spillover-Effekten zwischen firmeninternen Forschungsprojekten abgesehen (Cockburn/Henderson 2001; Henderson/Cockburn 1996), sind die Möglichkeiten, diese Risiken zu diversifizieren, begrenzt. Dementsprechend ist auch das aus dem CAPM Modell abgeleitete und durch das Beta ausgedrückte Marktrisiko für die Pharmaindustrie grösser als Eins. Für wachstumsstarke Unternehmen, deren Produkte sich noch im F\&E-Stadium befinden, ist das Beta sogar erheblich höher (Bernardo et al. 2007). Die vielen Marktaustritte in Form von Fusionen und Übernahmen in dieser Branche können vor diesem Hintergrund als Folge der mit der pharmazeutischen Forschung verbundenen Marktrisiken interpretiert werden. ${ }^{1}$ In der Studie von Danzon et al. (2007) wird dementsprechend gezeigt, dass eher Firmen mit schlecht gefüllten Produktpipelines übernommen

1 Die meisten Pharmaunternehmen sind an M\&A-Aktivitäten beteiligt und lediglich drei der „Big Pharma“Unternehmen in den USA waren in keine M\&A-Transaktion in den letzten 15 Jahren involviert (Danzon et al. 2004). Einige wichtige Fusionen und Übernahmen in der letzten Zeit waren Sanofi und Aventis, Glaxo, Wellcome, SmithKline French und Beecham, Roche, Chugai und Boehringer Mannheim, Pfizer und Warner Lambert. 
werden. Übernahmen durch andere Unternehmen stellen somit eine für diese Branche gängige Exit-Strategie dar. ${ }^{2}$

3. Intangibles Kapital: Neuere Erklärungsansätze betonen die Schwierigkeit, Gewinne und Kapital richtig zu messen und verweisen auf das von F\&E-Investitionen generierte intangible Kapital, welches nur unzureichend in einer Bilanz berücksichtigt wird (Salamon 1982; Salmi 1982; Taylor 1999; Mablich 2006). In den gängigen Rechnungslegungsvorschriften werden Forschungsinvestitionen im Rahmen der Gewinn- und Verlustrechnung als Aufwendungen ausgewiesen und nicht in der Bilanz als intangibles Vermögen aktiviert. Rentabilitätskennziffern wiederum setzen den Gewinn in Beziehung zum Kapital, entweder zum Eigenkapital (Eigenkapitalrentabilität - „return on equity“) oder zur Bilanzsumme eines Unternehmens (Gesamtkapitalrentabilität - „,return on total assets“). Werden die, intangibles Kapital generierenden, Forschungsinvestitionen aktiviert, so erhöht sich der im Nenner stehende Kapitalstock und die Rentabilität sinkt ceteris paribus. Allerdings gibt es noch einen gegenläufigen Effekt über die im Zähler stehenden Gewinne. Werden F\&E-Aufwendungen als Investitionen betrachtet, so müssen sie den Gewinnen hinzugerechnet werden. Lediglich die Abschreibungen auf das intangible Kapital wirken in diesem Fall gewinnmindernd. Welcher der beiden gegenläufigen Effekte dominiert, und ob die Aktivierung von F\&E-Investitionen die Rentabilität steigen oder sinken lässt, hängt dabei vom Wachstum der F\&E-Investitionen und dem Steuersatz ab (Lev et al. 2005).

In diesem Beitrag gehen wir der letzten Erklärungsmöglichkeit nach und argumentieren im Folgenden, dass die forschungsintensive Pharmaindustrie in einem besonderen Masse von einer Verzerrung der Rentabilitätskennziffern betroffen ist. Die forschenden Pharmaunternehmen in Deutschland gaben 200713 Prozent ihres Umsatzes für Forschung und Entwicklung aus; deutlich mehr als der Durchschnitt aller F\&E-betreibenden Unternehmen (4 Prozent) (VFA 2009, 25). Unter den 10 Unternehmen mit den höchsten Forschungsinvestitionen in Europa befinden sich mit GlaxoSmithKline, Sanofi-Aventis und AstraZeneca gleich drei Pharmaunternehmen, obwohl, gemessen an Umsatz und Beschäftigtenzahl, sie deutlich kleiner sind als die anderen Firmen in dieser Liga (European Commission 2006). Die F\&E-Investitionen der Pharmaindustrie weisen dabei einen sehr stark steigenden Verlauf seit den 1960er Jahren auf. Insbesondere in den 1990er Jahren hat sich diese Entwicklung verstärkt. Die durchschnittliche Wachstumsrate der F\&E-Investitionen zwischen 2001 und 2004 betrug laut einer EU-Studie 12,6 Prozent; damit lag sie mit Abstand an der Spitze der produzierenden Sektoren und deutlich über dem Durchschnitt aller Sektoren von 2,9 Prozent (European Commission 2006, 42).

Die empirische Literatur zu diesem Thema ist überschaubar. Werden intangibles Kapital generierende F\&E-Investitionen nicht als Aufwand behandelt, sondern gedanklich aktiviert, wächst der um F\&E-Investitionen korrigierte Kapitalstock den in Tabelle 1 aufgeführten Studien zufolge signifikant stärker als die Gewinne, so dass die Rentabilität von Pharmafirmen um bis zu sechs Prozentpunkte sinkt.

2 Dass Firmen, deren F\&E-Anstrengungen nicht mit einem neuen Produkt belohnt werden, schnell zu Übernahmekandidaten werden, betrifft selbst Grossunternehmen wie Astra, welches in den 90er Jahren das weltweit bestverkaufte Medikament Prilosec herstellte. In Ermangelung eines Prilosec Nachfolgepräparats fusionierte Astra schliesslich mit Zeneca. 
Mahlich/Yurtoglu | Intangibles Kapital und Rentabilität in der Pharmaindustrie

\begin{tabular}{|l|c|c|c|c|c|}
\hline & $\begin{array}{l}\text { Megna/Mueller } \\
(1991)\end{array}$ & $\begin{array}{l}\text { Grabowski } \\
\text { Mueller (1978) }\end{array}$ & $\begin{array}{l}\text { Clarkson } \\
(1977)\end{array}$ & Ayanian (1975) & Bloch (1974) \\
\hline Zeitraum & $1975-85$ & 1968 & $1965-74$ & 1973 & 1969 \\
\hline Stichprobe & 10 Firmen & 7 Firmen & 1 Firma & 6 Firmen & 4 Firmen \\
\hline $\begin{array}{l}\text { Gesamtkapital } \\
\text { Rentabilität }\end{array}$ & $14,8 \%$ & $14,1 \%$ & $17,3 \%$ & $17,7 \%$ & $9,7 \%-22,1 \%$ \\
\hline $\begin{array}{l}\text { Rentabilität bei } \\
\text { Aktivierung der } \\
\text { F\&E-Investitionen }\end{array}$ & $12,1 \%$ & $12,15 \%$ & $11,1 \%$ & $14,06 \%$ & $7,6 \%-16,1 \%$ \\
\hline $\begin{array}{l}\text { Differenz } \\
\text { (in Prozentpunkten) }\end{array}$ & 2,7 & 1,95 & 6,1 & 3,24 & $2,1-6,0$ \\
\hline
\end{tabular}

Tabelle 1: Rentabilität auf Bilanzsumme und korrigiertem Kapitalstock in der Pharmaindustrie

Megna/Mueller (1991) beispielsweise kommen zu dem Ergebnis, dass die unkorrigierte Gesamtkapitalrentabilität im gewichteten Durchschnitt um 2,7 Prozentpunkte höher ist als der korrigierte Wert, wobei Schering-Plough mit 4,9 Prozentpunkten die höchste und die mittlerweile in Sanofi-Aventis aufgegangene Firma Rorer mit 1 Prozentpunkten die niedrigste Differenz aufweisen. Die Ergebnisse bestätigen damit Resultate von älteren Untersuchungen, bei denen die festgestellte Differenz zwischen 1,95 und 6,1 Prozentpunkten liegt. Die beobachteten Unterschiede sind dabei auf die verschiedenen Untersuchungszeiträume sowie auf unterschiedliche Abschreibungsraten zurückzuführen.

Vor dem Hintergrund der bereits existierenden Literatur werden wir im folgenden empirischen Teil unseres Beitrages ebenfalls eine korrigierte Rentabilität errechnen, indem F\&EInvestitionen aktiviert und anschliessend abgeschrieben werden. In einem zweiten Schritt vergleichen wir die Rentabilität des um intangibles Kapital ergänzten Kapitalstocks der Pharmaindustrie mit der von anderen Branchen. Damit werden gleich mehrere Lücken in der Literatur geschlossen:

- Nach unserem Wissen ist die aktuellste empirische Untersuchung zu diesem Thema von Megna/Mueller (1991), die Daten aus den 70er und 80er Jahren verwendet. Unser Untersuchungszeitraum erstreckt sich demgegenüber von 1985 bis 2004.

- Die Stichprobengrösse der existierenden Untersuchungen ist recht klein und schwankt zwischen einer (Clarkson 1977) und zehn Firmen (Megna/Mueller 1991). Wir verwenden demgegenüber Daten von 86 Pharmaunternehmen und vergleichen sie mit denen von 3.296 Unternehmen anderer Branchen.

- Ebenfalls ist uns keine Arbeit bekannt, die mit nicht-US-amerikanischen Daten arbeitet. In unserer Stichprobe befinden sich hingegen Pharmaunternehmen aus neun verschiedenen Ländern. Es ist uns daher möglich, Vergleiche sowohl auf Branchen- als auch auf regionaler Ebene durchzuführen.

\section{Methodische Überlegungen}

\subsection{Bedeutung von intangiblem Kapital}

Mit dem Wandel von der Industrie- zur Wissensgesellschaft geht ein Wandel der unternehmerischen Wertschöpfung einher. In einer wissensbasierten Ökonomie verliert physisches Kapital in Form von Anlagen und Gebäuden für ein Unternehmen immer mehr an Bedeu- 
tung. An seine Stelle tritt intangibles Kapital in Form von Wissen, Beziehungen, Markenimage, Humankapital etc. ${ }^{3}$ Diese Kapitalarten werden durch die gängigen Rechnungslegungsvorschriften jedoch nur unzureichend erfasst und daher bleiben erfolgskritische Vermögenswerte in der Bilanz zum grossen Teil unsichtbar (Achleitner/Behr 2000). ${ }^{4}$ Allerdings sind in den letzten Jahren die Möglichkeiten erweitert worden, immaterielle Vermögensgegenstände in der Bilanz abzubilden, wobei hier unterschieden wird, ob sie aus Forschungs- oder aus Entwicklungsinvestitionen resultieren. Für Vermögensgegenstände, die aus Forschungsinvestitionen resultieren, gilt ein Aktivierungsverbot, da die Vermögensgegenstandseigenschaft von Forschungsergebnissen zu unsicher ist. Im Rahmen von IFRS können allerdings seit 2004 immaterielle Vermögenswerte angesetzt werden, die aus Entwicklungsinvestitionen resultieren (IAS 38). ${ }^{5}$ Laut IAS 38.8 muss ein immaterieller Vermögenswert identifizierbar in dem Sinne sein, dass er entweder separierbar ist und daher vom Unternehmen getrennt und verkauft oder vermietet werden kann, oder aber aus vertraglichen oder gesetzlichen Rechten entsteht, unabhängig davon, dass diese Rechte übertragbar sind. Der Deutsche Bundestag hat im März 2009 das Bilanzrechtsmodernisierungsgesetz (BilMoG) verabschiedet, demzufolge im Jahr 2010 das Aktivierungsverbot durch ein Ansatzwahlrecht für immaterielle Vermögensgegenstände für selbst geschaffene immaterielle Vermögensgegenstände des Anlagevermögens ersetzt wurde ( $\mathbb{2} 248$ BilMoG). Auch hier dürfen allerdings nur diejenigen Aufwendungen aktiviert werden, die bei der Entwicklung anfallen, während für Forschungsaufwendungen weiterhin ein Aktivierungsverbot besteht. Trotz der in letzter Zeit verbesserten rechtlichen Möglichkeiten, immaterielle Vermögensgegenstände zu aktivieren, erscheinen zumindest in der Pharmaindustrie kaum immaterielle Vermögenswerte in den Bilanzen, weshalb die Wirtschaftsprüfungsgesellschaft $P W C$ von einer Aktivierungsverweigerung spricht (PWC 2009).

Da intangibles Kapital in einer Bilanz nach wie vor weitgehend unberücksichtigt bleibt, wird eine Vielzahl von alternativen Methoden vorgeschlagen, den intangiblen Kapitalstock eines Unternehmens zu messen (siehe für einen Überblick Zambon et al. 2003). In der betriebswirtschaftlichen Praxis werden gerne Indikatorensysteme verwendet, bei denen immaterielles Vermögen durch unterschiedliche Kenngrössen aus verschiedenen Bereichen des Unternehmens erfasst wird. Bei dem von Edvinsson (1997) entwickelten „Scandia-Navigator" werden etwa Humankapital, Strukturkapital, Kundenkapital und Innovationskapital kategorisiert. Das Kundenkapital wiederum wird beispielsweise durch die Länge einer Kun-

3 Mayo (2001) unterscheidet drei Dimensionen des intangiblen Kapitals: 1. Kundenkapital (Beziehungen zu Kunden und Lieferanten, Marken, Reputation), 2. Strukturkapital (kodiertes Wissen in Form von Checklisten, dokumentierten Prozessen, Patenten), 3. Humankapital (Qualifikation der Mitarbeiter).

4 Während beim selbst geschaffenen („originären“) Geschäfts- oder Firmenwert (GoFW) Aktivierungsverbot besteht, kann der derivative GoFW, der im Rahmen einer Akquisition erworben wurde, Berücksichtigung in der Bilanz finden, wird allerdings in den unterschiedlichen Rechnungslegungsstandards unterschiedlich behandelt. Im HGB besteht beim derivativen GoFW ein Aktivierungswahlrecht. Bei Ausübung ist der GoFW nach $\ 255$ (4) HGB in jedem folgenden Geschäftsjahr zu mindestens einem Viertel durch Abschreibungen zu tilgen. Die Abschreibung des GoFW kann aber auch planmässig auf die Geschäftsjahre verteilt werden, in denen er voraussichtlich genutzt wird. Nach internationaler Rechnungslegung ist der Goodwill nach US-GAAP und IFRS/ IAS zwingend zu aktivieren und darf nicht planmässig abgeschrieben werden. Stattdessen ist seit dem Jahr 2002 ein jährlicher Werthaltigkeitstest („impairment test“) vorgeschrieben (SFAS 142 bzw. IAS 36), und eine Abschreibung ist nur dann erforderlich, wenn der Buchwert über dem Marktwert oder erzielbaren Betrag liegt.

5 Forschung ist nach IAS Definition die „eigenständige und planmässige Suche mit der Aussicht, zu neuen wissenschaftlichen oder technischen Erkenntnissen“ zu gelangen, während Entwicklung „die Anwendung von Forschungsergebnissen oder von anderem Wissen auf einen Plan oder Entwurf für die Produktion von neuen oder beträchtlich verbesserten Materialien, Vorrichtungen, Produkte, Verfahren, Systemen oder Dienstleistungen ist". (Amtsblatt der Europäischen Union L 392/1, 2004). 
denbeziehung oder die Zeit, die Mitarbeiter bei Kunden verbringen, gemessen. Auch der „Intangible Asset Monitor“ von Sveiby (1997), die „Balanced Scorecard“ von Kaplan/Norton (1992), der „Intellectual Capital Monitor“ von Steward (1997), das „Intellectual Capital Audit“ von Brooking (1996), die „Inclusive Value Methodology“ von M'Pherson/Pike (2001) oder das „Value Chain Scoreboard“ von Lev (2001) funktionieren nach ähnlichen Prinzipien. Gemein ist ihnen, dass sie lediglich Anhaltspunkte für die Qualität und Höhe des immateriellen Vermögens liefern, ohne weitere Hypothesen jedoch keine Verbindung zwischen Indikatorensystem und Geschäftserfolg hergestellt werden kann (Broda 2003). Ebenso wenig kann dem intangiblen Kapital ein in Geldeinheiten zu messender Wert zugeordnet werden.

Soll immaterielles Vermögen monetär bewertet werden, so ist man auf ökonomische Ansätze angewiesen. Das wohl am einfachsten zu beobachtende Mass in diesem Zusammenhang ist das Tobinsche $\mathrm{q}-\mathrm{d}$.h. der Quotient aus Marktwert und Wiederherstellungswert aller Vermögensgegenstände eines Unternehmens. Der Marktwert eines Unternehmens setzt sich aus der Summe des Marktwertes der Aktien und aus dem Marktwert des Fremdkapitals zusammen. Der Marktwert der Aktien (und auch des Fremdkapitals in Form von Anleihen) kann durch leicht zugängliche Börsenkurse bestimmt werden, weshalb diese Methode häufig in empirischen Arbeiten verwendet wird. Für den Wiederbeschaffungswert verwenden die meisten empirischen Studien den Buchwert des Gesamtkapitals. Übersteigt der Marktwert die Reproduktionskosten (q>1), so ist mit der Investition in den entsprechenden Vermögensgegenstand ein positiver Kapitalwert verbunden. Das Tobinsche q ist in der neoklassischen Investitionstheorie daher die entscheidende Determinante für den Erfolg einer Investition (Brainard/Tobin 1968; Hayashi 1982; Osterberg 1989). Im Rahmen dieses Ansatzes lässt sich der intangible Kapitalstock als Residualgrösse ermitteln, nämlich als Differenz aus dem Marktwert und den Wiederbeschaffungskosten.

Allerdings sind mit dem Rekurrieren auf den Marktwert und den Widerbeschaffungskosten verschiedene methodische Probleme verbunden. Einerseits hängt der Marktwert eines Unternehmens von weiteren Grössen ab, die von Unternehmen nicht beeinflusst werden können. $\mathrm{Zu}$ nennen wären hier beispielsweise konjunkturelle und makroökonomische Faktoren. Zum anderen unterstellt die dem Tobinschen q zugrunde liegende neoklassische Investitionstheorie, dass Kapitalmärkte den Wert eines Unternehmens richtig beurteilen. Gerade forschungsintensive Unternehmen scheinen an der Börse jedoch systematisch unterbewertet zu sein (Lev 2004). Unter Verwendung von Unternehmensdaten aus den USA, England, Frankreich, Deutschland und Italien zeigen Hall/Oriani (2006), dass das Ausmass der Unterbewertung in allen Ländern mit Ausnahme Englands eine ähnliche Grössenordnung annimmt. Ebenfalls schwierig ist die Festsetzung des Wiederbeschaffungswertes der Vermögensgegenstände, weil keine perfekten Märkte für „second hand assets“ existieren, obwohl empirisch arbeitende Autoren argumentieren, dass die Bilanzsumme sehr stark mit dem tatsächlichen Wiederbeschaffungswert korreliert (Perfect/Wiles 1994; Chung/Pruitt 1994; Lewellen/Badrinath 1997).

Aufgrund der mit der Tobinschen q Theorie verbundenen Schwierigkeiten verwenden wir im Folgenden die einleitend skizzierte Methode einer Aktivierung von F\&E-Investitionen mit anschliessender Abschreibung. Die Methode wird im nächsten Abschnitt detailliert erläutert. 


\subsection{Berechnung der Rentabilität unter Einbeziehung von intangiblem Kapital}

Bei der Berechnung der ökonomischen Rentabilität zum Zeitpunkt $\mathrm{t}$ (Пt) wird der operative Gewinn in Bezug zum Kapitalstock gesetzt. Da F\&E-Investitionen langfristige Effekte haben, die über den Zeitpunkt der ursprünglichen Ausgabe hinausgehen, werden sie dem Kapitalstock zugerechnet. Die Rentabilität ist daher folgendermassen definiert:

(1) $\Pi_{t}=\frac{S_{t}-V C_{t}-\lambda_{K} K_{t}-\lambda_{R} R_{t}}{K_{t}+R_{t}}$

Mit:

$S_{t}: \quad$ Umsatz zum Zeitpunkt $\mathrm{t}$

$V C_{t}$ : Variable Kosten zum Zeitpunkt t

$K_{t}:$ Tangibler Kapitalstock zum Zeitpunkt $\mathrm{t}$

$\lambda_{K}:$ Abschreibungsrate für den tangiblen Kapitalstock

$R_{t}$ : Intangibler Kapitalstock zum Zeitpunkt t, der durch frühere F\&E-Investitionen entstanden ist

$\lambda_{R}:$ Abschreibungsrate für den F\&E-Kapitalstock

Die am meisten verbreitete Annahme bezüglich des F\&E-Kapitalstocks ist, dass dieser linear abgeschrieben wird (Nerlove/Arrow 1962; Mansfield 1968; Schmalensee 1972; Grabowskil Mueller 1978; Hall/Oriani 2006). Auch wir verwenden eine lineare Abschreibung, da komplexere Abschreibungsraten zu keinen wesentlichen Veränderungen des berechneten Kapitalstocks führen (Nadiri/Prucha 1996). Die Annahme einer linearen Abschreibung impliziert die folgende Beziehung zwischen dem F\&E-Kapitalstock $\left(\mathrm{R}_{\mathrm{t}}\right)$ und den laufenden F\&E-Investitionen (rt):

(2) $R_{t}=r_{t}+\left(1-\lambda_{R}\right) R_{t-1}$

Wenn wir diese Gleichung rekursiv lösen, erhalten wir:

(3) $R_{t}=\sum_{k=0}^{\infty}\left(1-\lambda_{R}\right)^{k} r_{t-k}$

Die Gleichung (3) besagt, dass der F\&E-Kapitalstock ein gewichteter Durchschnitt der vergangenen F\&E-Investitionen ist, wobei die Gewichte in der Zeit exponentiell abnehmen.

Mit Gleichung (3) wird der unternehmensspezifische F\&E-Kapitalstock konstruiert. Als Basisszenario unterstellen wir eine Abschreibungsrate von $\lambda_{R}=10$ Prozent für den F\&E-Kapitalstock, eine Grössenordnung, die auch durch andere empirische Arbeiten gerechtfertigt wird (Baily 1972; Grabowski/Mueller 1978). Odagiri/Haneda (1998) beispielsweise verwenden eine Abschreibungsrate von 9,2 Prozent für den F\&E-Kapitalstock. Um die Belastbarkeit der Ergebnisse zu überprüfen, werden die Abschreibungsraten variiert; insbesondere werden höhere Abschreibungsraten angewendet, um die angesprochenen mit der Pharmaforschung verbundenen hohen Risiken besser abzubilden. Im folgenden Kapitel werden die Datenquellen und die empirischen Ergebnisse vorgestellt. 


\section{Empirische Untersuchung}

\subsection{Datenquellen}

Als Datengrundlage werden die Datenbanken Compustat und Compustat Global verwendet. Diese Datenbanken werden von der Firma Standard \& Poor's kommerziell zur Verfügung gestellt und beinhalten alle Variablen, die in Bilanzen sowie in Gewinn- und Verlustrechnungen vorkommen. Es handelt sich dabei jeweils um die konsolidierten Konzernabschlüsse börsennotierter Unternehmen. Der Untersuchungszeitraum umfasst die Jahre 1985 - 2004. Alle Zahlen wurden durch die jeweiligen Konsumentenpreisindices dividiert, um zu den realen, inflationsbereinigten Werten zu gelangen. In unserem Datensatz sind 3.382 börsennotierte Unternehmen enthalten. 86 davon sind aus der Pharmaindustrie und decken einen Grossteil des weltweiten Pharmamarktes ab. Der Pharmamarkt ist verhältnismässig stark konzentriert, und der Marktanteil der grössten 20 Unternehmen weltweit beträgt 61,5 Prozent (Srip 2009). Daher sind wir überzeugt, dass im Rahmen unserer Stichprobe - auch aufgrund des langen Zeitraumes - aussagekräftige und robuste Aussagen getroffen werden können.

\subsection{Darstellung der Methode anhand der Schering AG}

Anhand des deutschen Pharmaunternehmens Schering AG, welches im Jahr 2004 von der Bayer AG übernommen wurde, wird die in Kapitel 2.2 vorgestellte Untersuchungsmethode illustriert (Tabelle 2). Spalte (2) enthält die Bilanzsumme des Unternehmens („total assets“), also das sich im Zähler befindliche K aus Gleichung (1). Die variablen Kosten (VC) bestehen aus zwei Komponenten, die in den Spalten (3) und (4) wiedergegeben werden. In Spalte (3) befinden sich die so genannten „Cost of Good Sold“, also der Umsatzaufwand oder jene Kosten, die in einem unmittelbaren Zusammenhang mit den produzierten Waren stehen. ${ }^{6}$ In der Spalte (4) werden Vertriebskosten und allgemeine Verwaltungskosten aufgeführt. ${ }^{7}$ Spalte (5) beinhaltet die F\&E-Investitionen, also das r aus Gleichung (2). Spalte (6) enthält den Umsatz der Schering AG, entspricht also dem S in Gleichung (1). In der Spalte (7) schliesslich zeigen wir den durch die Abschreibungsrate von 10 Prozent abdiskontierten Wert der F\&E-Investitionen $\left(\mathrm{r}^{*}\right)$. Die F\&E-Investitionen des Jahres 1985 in Höhe von 421 Mio. EUR tragen mit 56,88 Mio. EUR zum intangiblen Kapitalstock des Jahres 2004 bei und werden anhand von Gleichung (3) ermittelt $\left[421 \times(1-0,9)^{19}\right]$. Für das Jahr 1986 ergibt sich dementsprechend ein $r^{*}$ von $500,54 x(0,1)^{18}=75,13$. In der nächsten Spalte (8) wird der abdiskontierte intangible Kapitalstock des jeweiligen Jahres berechnet. So ergibt sich der intangible Kapitalstock des Jahres 1986 in Höhe von 132,01 Mio. EUR aus der Summe der abdiskontierten F\&E-Investitionen aller Vorjahre (hier: 56,88 Mio. EUR) und den abdiskontierten F\&E-Investitionen des laufenden Jahres (hier: 75,13 Mio. EUR). Der für das Jahr 2004 berechnete Wert dieser Variablen $\left(\mathrm{R}_{2004}=6020,44\right.$ Mio. EUR) ist unser Mass für den intangiblen Kapitalstock der Schering AG.

6 Der Umsatzaufwand („Cost of Goods Sold“) entspricht den Produktionskosten, wenn es keine Lagerveränderungen gibt.

7 „Selling and General Expenses“. 


\begin{tabular}{|c|c|c|c|c|c|c|c|c|c|}
\hline (1) & (2) & (3) & (4) & (5) & (6) & (7) & (8) & (9) & (10) \\
\hline Jahr & $\begin{array}{l}\text { Bilanz- } \\
\text { summe }\end{array}$ & $\begin{array}{l}\text { COGS } \\
\text { Variable } \\
\text { Kosten } 2\end{array}$ & $\begin{array}{l}\text { SGA } \\
\text { Variable } \\
\text { Kosten } 1\end{array}$ & $\begin{array}{l}\text { F\&EE } \\
\text { Investi- } \\
\text { tionen }\end{array}$ & Umsatz & $\mathrm{r}^{*}$ & $\mathrm{Rt}$ & $\begin{array}{l}\text { Renta- } \\
\text { bilität } \\
\left(\Pi_{t}\right)\end{array}$ & $\begin{array}{l}\left.\Pi_{t}\right) \\
\text { Nach } \\
\text { Berück- } \\
\text { sichti- } \\
\text { gung } \\
\text { des } \\
\text { F\&E- } \\
\text { Kapitals }\end{array}$ \\
\hline 1985 & 2498,32 & & & 421,09 & 2522,92 & 56,88 & 56,88 & & \\
\hline 1986 & 3510,29 & 1467,14 & 0,00 & 500,54 & 2998,97 & 75,13 & 132,01 & 0,336 & 0,331 \\
\hline \multicolumn{10}{|l|}{$\cdots$} \\
\hline 1996 & 5053,80 & 636,47 & 1452,99 & 612,94 & 3406,70 & 263,85 & 1745,56 & 0,161 & 0,183 \\
\hline 1997 & 4513,36 & 614,18 & 1462,47 & 610,55 & 3426,17 & 292,02 & 2037,58 & 0,199 & 0,199 \\
\hline 1998 & 5014,49 & 616,43 & 1475,63 & 654,00 & 3418,01 & 347,56 & 2385,15 & 0,164 & 0,167 \\
\hline 1999 & 4244,88 & 675,27 & 1494,95 & 667,45 & 3585,13 & 394,13 & 2779,27 & 0,233 & 0,196 \\
\hline 2000 & 4325,73 & 695,96 & 1598,01 & 663,18 & 3674,06 & 435,11 & 3214,38 & 0,219 & 0,170 \\
\hline 2001 & 4033,42 & 738,25 & 1638,33 & 665,80 & 3731,23 & 485,37 & 3699,75 & 0,236 & 0,161 \\
\hline 2002 & 4794,57 & 716,02 & 1758,00 & 758,42 & 4022,72 & 614,32 & 4314,06 & 0,223 & 0,153 \\
\hline 2003 & 5639,58 & 874,35 & 1963,77 & 867,78 & 4534,22 & 781,00 & 5095,06 & 0,201 & 0,138 \\
\hline 2004 & 6149,23 & 913,29 & 2086,37 & 925,37 & 4941,03 & 925,37 & 6020,44 & 0,216 & 0,135 \\
\hline
\end{tabular}

Tabelle 2: Rentabilität der Schering AG [1985 bis 2004]. Spalten (1) - (8) in Mio. EUR

Die Schering AG hat in unserem Beobachtungszeitraum eine sehr F\&E-intensive Strategie gewählt, die durch eine F\&E-Quote von durchschnittlich 17 Prozent des Umsatzes reflektiert wird. Aufgrund der hohen F\&E-Investitionen ist auch der resultierende F\&E-Kapitalstock hoch und entspricht in etwa dem Wert der Bilanzsumme. Insofern ist es zu erwarten, dass eine Berücksichtigung des F\&E-Kapitalstocks zu einer starken Revision der in Spalte (9) aufgeführten Rendite auf die Bilanzsumme in Höhe von 21,6 Prozent führt. Tatsächlich beobachten wir in Spalte (10), dass die Rentabilität unter Berücksichtigung des F\&E-Kapitalstocks auf einen Wert von lediglich 13,5 Prozent sinkt. 


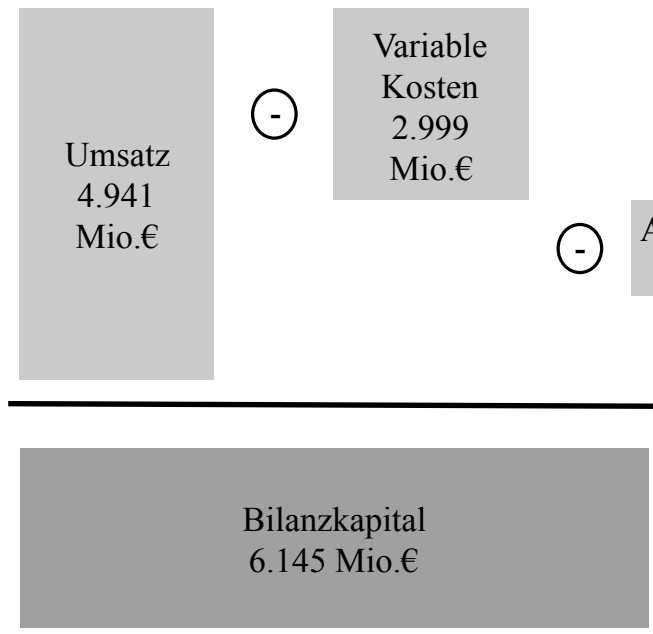

Abbildung 1: Rentabilität der Schering AG [2004]

Der Anschaulichkeit halber wird der eben erläuterte Zusammenhang in den Abbildungen 1 und 2 grafisch illustriert. Abbildung 1 stellt die Rentabilität ohne Aktivierung der F\&E-Investitionen dar, bei dem als Basis die in der Bilanz ausgewiesene Bilanzsumme verwendet wird. Bei der Aktivierung der F\&E-Investitionen müssen die laufenden F\&E-Investitionen von 925 Mio. EUR wieder von den variablen Kosten abgezogen werden, was den Gewinn erhöht (Abbildung 2). Allerdings kommen nun zusätzliche Abschreibungen auf den F\&EKapitalstock von 602 Mio. EUR dazu, die wiederum den Gewinn mindern. Als Nettoeffekt ermitteln wir im Vergleich zu Abbildung 1 dennoch einen höheren Gewinn in Höhe von 1.650 Mio. EUR. Als Basis steht im Nenner die Summe aus Bilanzsumme (6.145 Mio. EUR) und F\&E-Kapitalstock (6.020 Mio. EUR). Da der Nenner weitaus stärker wächst als der im Zähler stehende Gewinn, kommt es zu einer Verringerung der Rentabilität.

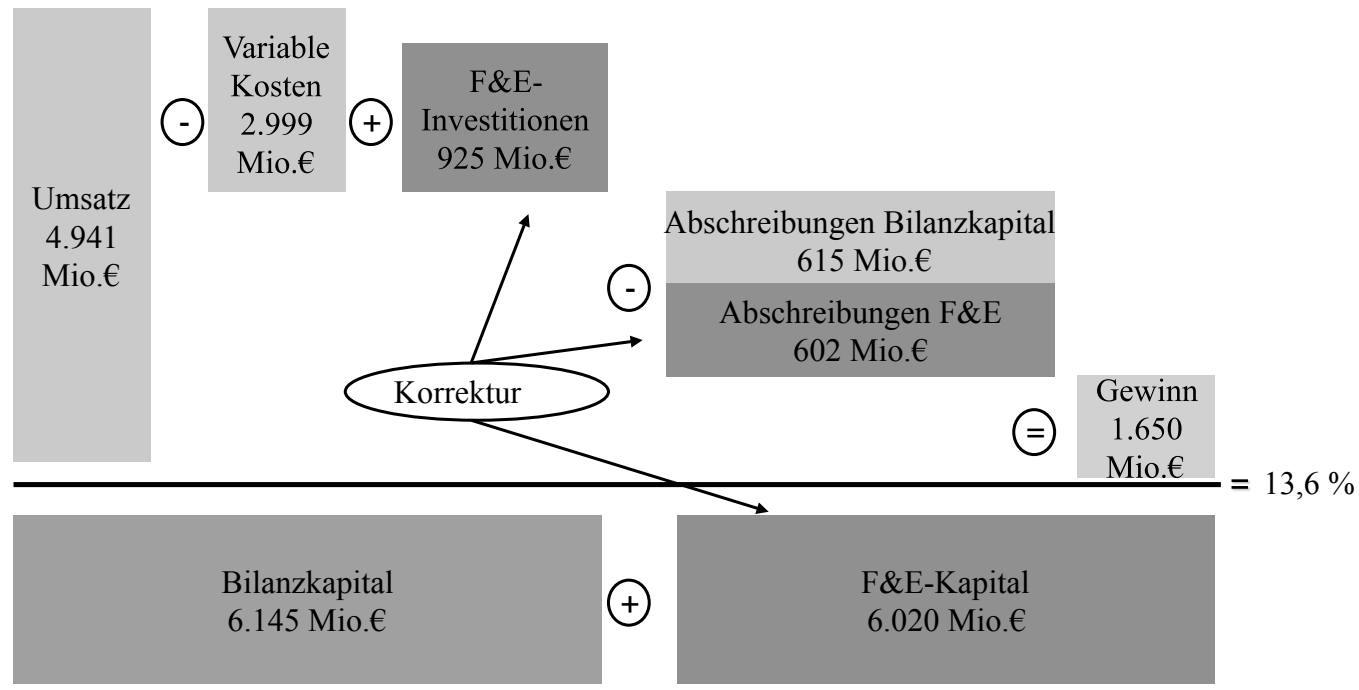

Abbildung 2: Korrigierte Rentabilität der Schering AG [2004] 


\subsection{Resultate für Pharma-Unternehmen aus Europa, den USA und Japan}

In diesem Abschnitt erweitern wir unsere Analyse auf 86 Pharmaunternehmen aus Europa, den USA, Japan und dem Rest der Welt. Tabelle 3 enthält eine Aufstellung der Rentabilität (arithmetisches Mittel und Median) ohne und mit Berücksichtigung des F\&E-Kapitalstocks.

Signifikant unter dem Gesamtdurchschnitt liegt die Rentabilität in Japan. Dies wird in der Regel auf die Wachstumspräferenz japanischer Firmen zurückgeführt, da sich das japanische Management den Anteilseignern weniger verpflichtet fühlen als den Mitarbeitern (Odagiri 1992; Aoki 1990). Eine signifikant überdurchschnittliche Rentabilität hingegen weisen Firmen aus dem „Rest der Welt“ auf. Hier spielt die Tatsache eine Rolle, dass die Pharmaindustrie in vielen „emerging markets“ politisch von ausländischem Wettbewerb geschützt wird.

Es ist weiterhin ersichtlich, dass es in allen Regionen zu einem erheblichen Rückgang der buchhalterischen Renditekennzahlen kommt. Der durchschnittliche Rückgang der Rentabilität unter Einbeziehung des F\&E-Kapitalstocks beträgt in Europa über 5,3 Prozentpunkte. Etwas geringere Diskrepanzen von um die 2 Prozentpunkte sind auch in den USA und um die 3,2 Prozentpunkte in Japan festzustellen. Die hohe Diskrepanz der Rentabilität zwischen Europa und den USA könnte mit den unterschiedlichen Rechnungslegungsvorschriften erklärt werden, da der Umgang mit dem derivativen Firmenwert in den USA weniger restriktiv als in Europa oder Japan ist. Nach US-GAAP ist der Firmenwert („goodwill“) nicht zwingend abzuschreiben, sondern einem regelmässigen „impairment test“ zu unterziehen. Allerdings ist einschränkend zu erwähnen, dass der „Impairment Only Approach“ in den USA erst 2001 eingeführt wurde und daher unsere Ergebnisse wahrscheinlich nicht entscheidend beeinflusst. Eine weitere Erklärung ist, dass die europäischen Pharmafirmen im Vergleich zu amerikanischen forschungsintensiver sind und daher einen relativ höheren F\&E-Kapitalstock aufweisen.

\begin{tabular}{|c|c|c|c|c|c|c|c|}
\hline & $\begin{array}{l}\text { Anzahl } \\
\text { Unterneh- } \\
\text { men }\end{array}$ & $\begin{array}{l}\text { Durch- } \\
\text { schnitt } \\
\Pi_{t}(\%)\end{array}$ & $\begin{array}{c}\text { Durch- } \\
\text { schnitt } \\
\Pi_{t}(F \& E) \\
(\%)\end{array}$ & Differenz & $\begin{array}{c}\text { Median } \\
\Pi_{t}(\%)\end{array}$ & $\begin{array}{c}\text { Median } \\
\Pi_{t}(F \& E) \\
(\%)\end{array}$ & Differenz \\
\hline Europa & 21 & 11,09 & 5,75 & 5,34 & 13,04 & 8,49 & 4,55 \\
\hline USA & 27 & 8,69 & 6,64 & 2,05 & 7,93 & 4,96 & 2,97 \\
\hline Japan & 22 & $3,67 * *$ & $0,38 * * *$ & 3,29 & 3,72 & 0,17 & 3,55 \\
\hline Rest & 16 & $23,72 * * *$ & $20,53 * *$ & 3,19 & 5,52 & 2,75 & 2,77 \\
\hline Gesamt & 86 & 10,79 & 7,41 & 3,38 & 5,86 & 0,40 & 5,46 \\
\hline
\end{tabular}

Tabelle 3: Überblick der empirischen Ergebnisse für Pharmaunternehmen

Wie aus Tabelle 4 hervorgeht, beträgt der Anteil des F\&E-Kapitalstocks (R) am gesamten Kapitalstock $(\mathrm{R}+\mathrm{K})$ für die europäischen Unternehmen in unserer Stichprobe um die 42 Prozent, für japanische Firmen 35 Prozent und für amerikanische lediglich 25 Prozent. Dieses Ergebnis mag manche Marktbeobachter insofern verblüffen, als amerikanische Pharmafirmen aufgrund ihrer Forschungserfolge und erfolgreichen Innovationen in den letzten 1520 Jahren die Weltmarktführerschaft erlangt haben. Nicht mehr Deutschland ist die „Apotheke der Welt", wie es einmal in den 1980er Jahren hiess, sondern die USA. Über zwei Drittel der weltweit meistverkauften Medikamente stammen bereits aus den USA. Die 
hohe Innovationskraft amerikanischer Unternehmen in Kombination mit einem relativ geringeren Einsatz an F\&E-Kapital spricht dafür, dass amerikanische Unternehmen ihre Ressourcen produktiver einsetzen als europäische Unternehmen.

\begin{tabular}{|l|c|c|c|}
\hline & Durchschnitt (\%) & Median (\%) & Standardabweichung (\%) \\
\hline Europa & 41,55 & 38,07 & 19,04 \\
\hline USA & 25,64 & 24,92 & 9,15 \\
\hline Japan & 34,61 & 35,54 & 7,73 \\
\hline Rest & 34,99 & 20,57 & 33,59 \\
\hline Total & 33,56 & 31,54 & 19,00 \\
\hline
\end{tabular}

Tabelle 4: Anteil des F\&E-Kapitalstocks am gesamten Kapitalstock

Die Resultate basieren auf einer angenommenen Abschreibungsrate für den F\&E-Kapitalstock von 10 Prozent. Um die Sensitivität dieses Ergebnisses zu überprüfen, haben wir die Resultate mit zwei zusätzlichen Abschreibungsraten neu kalkuliert. Bei einer Abschreibungsrate von 5 Prozent beträgt die durchschnittliche korrigierte Rentabilität 9,54 Prozent und ist somit 1,24 Prozentpunkte niedriger als die Rentabilität auf Bilanzaktiva. Bei einer Abschreibungsrate von 7,5 Prozent liegt die Diskrepanz bei 2,00 Prozentpunkten. Auch die Ergebnisse aus Tabelle 4 sind robust gegenüber Änderungen der Abschreibungsraten. Der durchschnittliche Anteil variiert zwischen 36 Prozent bei einer fünfprozentigen Abschreibungsrate und 38,7 Prozent bei einer Abschreibungsrate von 7,5 Prozent. ${ }^{8}$

\subsection{Vergleich mit anderen Branchen}

Die zweite Frage, die sich in diesem Zusammenhang stellt, ist, ob die Korrektur der Rentabilität eine Nivellierung im Vergleich mit anderen Branchen zur Folge hat, oder ob Pharmafirmen auch unter Einbeziehung des intangiblen Kapitalstocks besonders gut verdienen. Um diese Frage zu beantworten, muss die korrigierte Rentabilität mit jener von anderen Sektoren verglichen werden. In der Literatur wurde bereits gezeigt, dass die Differenz zwischen der korrigierten und der unkorrigierten Rentabilität bei den Pharmafirmen im Schnitt grösser als bei Firmen anderer Branchen ist. Dementsprechend schrumpft der Rentabilitätsvorsprung in den Pharmafirmen je nach Studie auf bis zu 0,7 Prozentpunkte zusammen (Megna/Mueller 1991). Bei älteren Arbeiten beträgt der Unterschied der Rentabilität zwischen 3,3 Prozent (Clarkson 1977) und 3,6 Prozent (Grabowski/Mueller 1978). Anzumerken ist zudem, dass die erwähnten Untersuchungen Daten aus den 1970er und 1980er Jahren verwendet haben. In der Zwischenzeit dürfte sich der Abstand aufgrund der eingangs erwähnten hohen Dynamik bei den F\&E-Investitionen weiter verringert haben.

In der folgenden Tabelle 5 präsentieren wir den Rentabilitätsvergleich zwischen den unterschiedlichen Branchen nach „Standard Industrial Classification“-Zweistellern, in dem wir bei allen Branchen die F\&E-Investitionen gedanklich aktiviert haben. Die „Standard Industrial Classification“ (SIC) ist ein auf Unternehmensbefragungen beruhendes Klassifikationsschema für Industriebranchen. Die Unternehmen ordnen sich dabei aufgrund ihres Hauptproduktes selber einer Branche zu, wobei die Branchen je nach Aggregationsstufe weiter aufgegliedert werden können in zwei, drei- oder vierstellige Branchencodes. Aus der

8 Die vollständige regionenspezifische Auswertung ist auf Anfrage von den Autoren erhältlich. 
Tabelle 5 ist ersichtlich, dass die Pharmaindustrie mit einer durchschnittlichen Differenz zwischen unkorrigierter und korrigierter Rentabilität von 3,38 Prozentpunkten knapp nach den sehr heterogen definierten unternehmensnahen Dienstleistungen („Business Services“) und der Land- und Forstwirtschaft den dritten Rang aller Branchen einnimmt. Die durchschnittliche Korrektur der Rentabilität beträgt über alle Sektoren hingegen lediglich 1,88 Prozentpunkte. Bei den ebenfalls F\&E-intensiven Branchen Elektronik oder Chemie beträgt die Korrektur 3,26 bzw. 2,30 Prozentpunkte.

Noch augenfälliger ist dieser Effekt bei der Betrachtung der gegenüber Ausreissern weniger empfindlichen Mediane. Die Rentabilität der Pharmaindustrie ist hier mit 5,86 Prozent fast fünfmal so hoch wie der Median aller Branchen (1,28 Prozent). Von 35 betrachteten Branchen liegt die Pharmaindustrie nach der Öl- und Gasförderung, der Mineralölverarbeitung und den Verlags- und Druckerzeugnissen an fünfter Stelle. Nach Korrektur und der Aktivierung der F\&E-Investitionen fällt die Rentabilität auf 0,4 Prozent und liegt damit nur noch knapp über der aller Branchen (0,3 Prozent). Die Pharmaindustrie fällt dadurch auf den 18ten Rang zurück.

\begin{tabular}{|l|r|r|r|r|r|r|r|r|}
\hline & & \multicolumn{2}{|c|}{$\Pi_{\mathbf{t}}(\%)$} & \multicolumn{2}{|c|}{$\Pi_{\mathbf{t}}(\mathrm{F} \& \mathrm{E})(\%)$} & & \multicolumn{2}{|c|}{$\begin{array}{c}\text { Anteil intangibles } \\
\text { Kapital am Ge- } \\
\text { samtkapital }(\%)\end{array}$} \\
\hline Branche & $\mathrm{N}$ & $\begin{array}{c}\text { Arithm. } \\
\text { Mittel }\end{array}$ & Median & $\begin{array}{c}\text { Arithm. } \\
\text { Mittel }\end{array}$ & Median & Diff. & $\begin{array}{c}\text { Arithm. } \\
\text { Mittel }\end{array}$ & Median \\
\hline $\begin{array}{l}\text { Unternehmensbezogene } \\
\text { Dienstleistungen }\end{array}$ & 122 & 9,87 & 1,33 & 5,92 & $-0,82$ & 3,95 & 28,69 & 20,05 \\
\hline $\begin{array}{l}\text { Land-, Forstwirtschaft, } \\
\text { Fischerei }\end{array}$ & 38 & 1,92 & $-2,72$ & $-1,93$ & $-3,17$ & 3,85 & 15,52 & 9,13 \\
\hline Pharmazeutika & 86 & 10,79 & 5,86 & 7,41 & 0,40 & 3,38 & 33,56 & 31,54 \\
\hline $\begin{array}{l}\text { Geräte der Elektrizitäts- } \\
\text { erzeugung und -verteilung }\end{array}$ & 262 & 6,14 & 0,65 & 2,88 & $-0,77$ & 3,26 & 24,86 & 21,85 \\
\hline $\begin{array}{l}\text { Medizin-, Mess-, Steuer-, } \\
\text { Regelungstechnik; Optik }\end{array}$ & 134 & 6,09 & 2,81 & 3,03 & 1,53 & 3,06 & 25,08 & 24,54 \\
\hline Textilien & 66 & 7,14 & $-2,03$ & 4,20 & $-3,05$ & 2,94 & 13,16 & 11,46 \\
\hline Maschinenbau, Computer & 231 & 8,36 & 0,79 & 5,63 & $-0,33$ & 2,73 & 19,87 & 15,95 \\
\hline Fahrzeugbau & 128 & 9,77 & 1,69 & 7,16 & 0,25 & 2,61 & 14,11 & 14,22 \\
\hline Andere Dienstleistungen & 197 & 8,14 & 0,56 & 5,80 & 0,09 & 2,34 & 6,86 & 1,08 \\
\hline Stahl- und Leichtmetallbau & 89 & 12,82 & 1,43 & 10,51 & 0,25 & 2,31 & 9,47 & 7,62 \\
\hline Chemische Erzeugnisse & 199 & 8,40 & 2,02 & 6,10 & 0,54 & 2,30 & 15,80 & 15,16 \\
\hline Möbel und Beschläge & 29 & 22,21 & 3,73 & 20,32 & 3,06 & 1,89 & 11,97 & 10,04 \\
\hline Verschiedenes & 150 & 3,55 & 1,13 & 1,67 & 0,62 & 1,88 & 29,34 & 26,12 \\
\hline Verlags- und Druckerzeugnisse & 71 & 17,28 & 6,10 & 15,56 & 4,57 & 1,72 & 8,21 & 6,03 \\
\hline Öl- und Gasförderung & 68 & 8,02 & 8,30 & 6,34 & 6,92 & 1,68 & 26,40 & 29,54 \\
\hline Steine, Erde, Glas, Keramik & 83 & 13,25 & 2,82 & 11,65 & 1,88 & 1,60 & 6,32 & 5,01 \\
\hline Nahrungs- und Genussmittel & 194 & 13,02 & 2,19 & 11,50 & 1,49 & 1,52 & 7,41 & 5,35 \\
\hline Gummi, Kunststoffwaren & 66 & 2,63 & 0,97 & 1,13 & 0,15 & 1,50 & 12,35 & 11,58 \\
\hline Nachrichtenübermittlung & 63 & 11,13 & 5,06 & 9,79 & 4,36 & 1,34 & 7,31 & 4,62 \\
\hline Erzbergbau & 57 & 3,74 & $-0,97$ & 2,50 & $-0,95$ & 1,24 & 9,71 & 2,49 \\
\hline Tabakverarbeitung & 10 & 21,05 & 7,70 & 19,83 & 6,97 & 1,22 & 5,32 & 5,22 \\
\hline Papier, Pappe & 70 & 10,14 & $-0,39$ & 8,95 & $-0,52$ & 1,19 & 7,66 & 4,23 \\
\hline Diverse Sachgütererzeugung & 37 & 1,60 & 0,48 & 0,43 & $-0,41$ & 1,17 & 16,60 & 14,70 \\
\hline
\end{tabular}


Mahlich/Yurtoglu | Intangibles Kapital und Rentabilität in der Pharmaindustrie

\begin{tabular}{|l|r|r|r|r|r|r|r|r|}
\hline Kleidung & 39 & 11,59 & 1,36 & 10,43 & 0,96 & 1,16 & 5,61 & 3,66 \\
\hline Holz, Kork, Flechtwaren & 27 & 11,88 & 0,23 & 10,77 & $-0,02$ & 1,11 & 6,41 & 3,88 \\
\hline $\begin{array}{l}\text { Grosshandel } \\
\text { (dauerhafte Güter) }\end{array}$ & 117 & 7,66 & $-1,24$ & 6,57 & $-1,62$ & 1,09 & 5,20 & 2,49 \\
\hline Leder und Ledererzeugnisse & 14 & 2,57 & 3,37 & 1,50 & 2,59 & 1,07 & 15,27 & 14,17 \\
\hline $\begin{array}{l}\text { Metallerzeugung und } \\
\text { Bearbeitung }\end{array}$ & 115 & 15,64 & 1,90 & 14,74 & 1,64 & 0,90 & 8,33 & 5,51 \\
\hline Bau & 158 & 5,63 & $-4,13$ & 4,83 & $-4,72$ & 0,80 & 3,90 & 2,18 \\
\hline Grosshandel (Konsumgüter) & 66 & 3,46 & $-1,78$ & 2,74 & $-2,07$ & 0,72 & 6,61 & 1,31 \\
\hline Energie- und Wasserversorgung & 68 & 13,00 & 3,81 & 12,33 & 3,36 & 0,67 & 4,37 & 1,81 \\
\hline $\begin{array}{l}\text { Kokerei, Mineralöl- } \\
\text { verarbeitung, }\end{array}$ & 37 & 7,98 & 6,87 & 7,35 & 6,73 & 0,63 & 5,56 & 3,57 \\
\hline Verkehr & 116 & 12,10 & $-0,65$ & 11,66 & $-0,68$ & 0,44 & 4,63 & 0,66 \\
\hline Einzelhandel & 248 & 11,72 & 2,41 & 11,42 & 2,35 & 0,30 & 1,38 & 0,04 \\
\hline $\begin{array}{l}\text { Gewinnung von Steinen und } \\
\text { Erden }\end{array}$ & 13 & 1,00 & 2,87 & 0,97 & 2,91 & 0,03 & 6,94 & 3,78 \\
\hline Gesamt & 3.382 & 9,03 & 1,28 & 7,15 & 0,30 & 1,88 & 12,83 & 7,04 \\
\hline
\end{tabular}

Tabelle 5: Rentabilitätsvergleich aller Sektoren weltweit

\section{Fazit}

Die auf den ersten Blick hohe Rentabilität der Pharmaindustrie kann zu einem wesentlichen Teil auf die Vernachlässigung des intangiblen Kapitals in der konventionellen Rechnungslegung zurückgeführt werden. Die von uns berechnete korrigierte Rentabilität, bei der F\&EInvestitionen nicht als Aufwand, sondern als Investitionen bewertet werden, die über einen längeren Zeitraum abgeschrieben werden, zeigt, dass die so errechnete Rentabilität bei europäischen Unternehmen im Durchschnitt etwa 4 bis 7 Prozentpunkte unter der Rentabilität auf die Bilanzsumme liegt. Der Vorsprung der Pharmaindustrie bezüglich ihrer Rentabilität im Vergleich mit anderen Branchen wird dadurch fast vollständig nivelliert. Würden die in dieser Arbeit nicht berücksichtigten Werbeinvestitionen, die ebenfalls intangibles Kapital in Form von Markenwert generieren, hinzugerechnet, so würde sich die Rentabilität der sehr werbeintensiven Pharmaindustrie möglicherweise weiter reduzieren. Die Pharmaindustrie betreffende Kostendämpfungsmassnahmen im Gesundheitswesen wie Zwangsrabatte für verschreibungspflichtige patentgeschützte Arzneimittel oder Festpreise für Medikamente sollten ebenfalls im Lichte dieser Ergebnisse gesehen werden, zumal F\&EInvestitionen sehr stark auf institutionelle Anreize und erwartete Erträge reagieren (Mahlich 2005; Mahlich/Roediger-Schluga 2006; Grabowksi/Vernon 2000).

Inwieweit aus unseren Ergebnissen die praktische Forderung abgeleitet werden kann, die Aktivierung von F\&E-Investitionen der Transparenz wegen verpflichtend durchzuführen, hängt von der entsprechenden Rechnungslegungsphilosophie ab. Verfechter von gläubigerorientierten Systemen mögen argumentieren, dass in Personen gebundene intangible Vermögensgegenstände von den Gläubigern im Zweifelsfalle nicht veräussert werden können, weshalb Liquidationskosten und F\&E-Investitionen positiv korrelieren (Alderson/Betker 1996). Eine verpflichtende Aktivierung würde zwar zusätzliches Vermögen ausweisen, auf das der Gläubiger aber im Insolvenzfall so gut wie keinen Zugriff hat. Befürworter einer stärkeren Investorenorientierung im Rechnungswesen hingegen mögen einen solchen Schritt befürworten, da er zu einem tieferen Verständnis des entsprechenden Unternehmens beiträgt. 
Daher würde es unserer Meinung nach reichen, wenn die entsprechenden Werte in einem Anhang ausgewiesen werden.

Abschliessend soll auf einige Einschränkungen unserer Arbeit hingewiesen werden. Leider können wir in unserer Analyse nicht zwischen Forschungs- und Entwicklungsinvestitionen unterscheiden, da diese von den Unternehmen nicht getrennt berichtet werden. Diese Unterscheidung wäre insofern wichtig, als dass Forschungsinvestitionen aufgrund ihres hohen Risikos mit einer höheren Abschreibungsrate versehen werden müssten als die marktnahen Entwicklungsprojekte. Weiterhin erschwert die in unserem Untersuchungszeitraum eingesetzte Harmonisierung der unterschiedlichen Rechnungslegungsstandards ein wenig die Vergleichbarkeit der Ergebnisse. Zukünftige Arbeiten zu diesem Thema sollten daher versuchen, diese nach wie vor bestehende Forschungslücke zu schliessen.

\section{Literaturhinweise}

Achleitner, A.-K/Behr, G. (2000): International Accounting Standards, München.

Adams, C.P./Brantner, Van V. (2006): Estimating the cost of new drug development: is it really 802 million dollars?, in: Health Affairs, Jg. 25, S. 420-428.

Alderson, M.J./Betker, B.L. (1996): Liquidation Costs and Accounting Data, in: Financial Management, Jg. 25, S. 25-36.

Angell, M. (2004): The Truth About the Drug Companies: How They Deceive Us and What To Do About It, New York.

Aoki, M. (1990): Toward an Economic Model of the Japanese Firm, in: Journal of Economic Literature, Jg. 28, S. 1-27.

Ayanian, R. (1975): Advertising and Rate of Return, in: Journal of Law and Economics, Jg. 18, S. 479-506.

Baily, M.N. (1972): Research and Development Costs and Returns: The U.S. Pharmaceutical Industry, in: Journal of Political Economy, Jg. 80, S. 70-85.

Bain, J.S. (1951): Relation of Profit Rate to Industry Concentration: American Manufacturing, 19361949, in: Quarterly Journal of Economics, Jg. 65, S. 293-324.

Bain, J.S. (1956): Barriers to New Competition, Cambridge.

Bernardo, A.E./Chowdhry, B./Goyal, A. (2007): Growth Options, Beta, and the Cost of Capital, in: Financial Management, Jg. 36, S. 5-18.

Bloch, H. (1974): Advertising and Profitability: A Reappraisal, in: Journal of Political Economy, Jg. 82, S. 267-286.

Brainard, W./Tobin, J. (1968): Pitfalls in financial model building', in: American Economic Review, Jg. 8, Nr. 2, S. 99-122.

Broda, B. (2003): Alternative Ansätze zur Messung des intellektuellen Kapitals, in: Der Schweizer Treuhänder, Nr. 09, S. 729-740.

Brooking, A. (1996), Intellectual Capital: Core Asset for the New Millennium, London.

Chung, K.H./Pruitt, S.W. (1994): A simple approximation of Tobins q, in: Financial Management, Jg. 23, S. 70-74.

Clarkson, K. W. (1977): Intangible Capital and Rates of Return, Washington.

Cockburn, I.M./Henderson, R.M. (2001): Scale and Scope in Drug Development: Unpacking the Advantage of Size in Pharmaceutical Research, in: Journal of Health Economics, Jg. 20, S. 10331057.

Comanor, W.S./Wilson, T.A. (1967): Advertising, Market Structure and Performance, in: The Review of Economics and Statistics, Jg. 49, S. 423-440. 
Danzon, P.M./Epstein, A./Nicholson, S. (2007): Mergers and Acquisitions in the Pharmaceutical and Biotech Industries, in: Managerial and Decision Economics, Jg. 28, S. 307-328.

Die Weltwoche (2004): Im Wesen nichts Neues, Interview mit Marcia Angell, Ausgabe 37/04 vom 08.09.2004.

DiMasi, J.A./Hansen, R.W./Grabowski, H.G. (2003): The Price of Innovation: New Estimates of Drug Development Costs, in: Journal of Health Economics, Jg. 22, S. 151-185.

Domowitz, I./Hubbard, G./Peterson, B. (1986): Business Cycles and the Relationship between PriceCost-Margins, in: Rand Journal of Economics, Jg. 17, S. 1-17.

Edvinsson, L. (1997): Intellectual Capital, Stockholm.

European Commission (2006), The 2005 EU Industrial R\&D Investment Scoreboard, DirectorateGeneral Joint Research Centre, Institute for Prospective Technological Studies, Luxemburg.

Fortune (2010): FORTUNE 500: Our annual ranking of America's largest corporations, unter: http:// money.cnn.com/magazines/fortune/fortune500_archive/full/1955/, abgerufen am 20.4.2010.

Grabowski, H.G./Mueller, D.C. (1978): Industrial Research and Development, Intangible Capital Stocks, and Firm Profit Rates, in: The Bell Journal of Economics, Jg. 9, S. 328-343.

Grabowski, H.G./Vernon, J./DiMasi, J. (2003): Returns on Research and Development for 1990's New Drug Introductions, in: PharmacoEconomics, Jg. 20 (3), S. 11-29.

Grabowski H.G./Vernon, J. (2000): The determinants of pharmaceutical research and development expenditures, in: Journal of Evolutionary Economics, Jg. 10, S. 201-215.

Grabowski, H.G./Vernon, J. (1990): A new look at the returns and risks to pharmaceutical R\&D, in: Management Science, Jg. 36, Nr. 7, S. 804-821.

Gschwandtner, A./Cable, J. (2008): On modeling the persistence of profits in the long run: An analysis of 156 US companies, 1950-1999, in: International Journal of the Economics of Business, Jg. 15, S. 245-263.

Hall, B./Oriani, R. (2006): Does the Market Value R\&D Investment by European Firms? Evidence from a Panel of Manufacturing Firms in France, Germany, and Italy, in: International Journal of Industrial Organization, Jg. 24, Nr. 5, S. 971-993.

Hayashi, F. (1982): Tobin's Marginal q and Average q: A Neoclassical Interpretation, in: Econometrica, Jg. 50, S. 213-224.

Henderson, R./Cockburn, I. (1996): Scale, scope and spillovers: the determinants of research and productivity in drug industry, in: RAND Journal of Economics, Jg. 27, S. 32-59.

Informa Healthcare (2009): Scrip Pharmaceutical League Tables 2009, London.

Kaplan, R.S./Norton, D.P. (1992): The Balanced Scorecard- Measures that Drive Performance, in: Harvard Business Review, Jg. 70, S. 71-79.

Lev, B. (2001): Intangibles: Management, Measurement, and Reporting, Washington.

Lev, B. (2004): Sharpening the Intangible Edge, in: Harvard Business Review, Jg. 82, S. 109-116.

Lev, B./Sarath, B./Sougiannis, T. (2005): R\&D Reporting Biases and their Consequences, in: Contemporary Accounting Research, Jg. 22, S. 977-1026.

Lewellen, G. Wilbur/Badrinath, S-G. (1997): On the Measurement of Tobins q, in: Journal of Financial Economics, Jg. 44, S. 77-122.

Mablich, J. (2006): Anmerkungen zur Profitabilität der Pharmaindustrie, in: Wirtschaftswissenschaftliches Studium, Jg. 35, Nr. 2, S. 73-81.

Mablich, J. (2005): Wirtschaftspolitik für wissensintensive Branchen am Beispiel der Pharmaindustrie, in: Zeitschrift für Wirtschaftspolitik, Jg. 54, S. 366-387.

Mahlich, J./Roediger-Schluga, T. (2006): Determinants of Pharmaceutical R\&D: Evidence from Japan, in: Review of Industrial Organization, Jg. 28, S. 145-164. 
M'Pherson, P.K./Pike, S. (2001): Accounting, Empirical Measurement and Intellectual Capital, in: Journal of Intellectual Capital, Jg. 2, S. 246-260.

Mansfield, E. (1968): The Economics of Technological Change, New York.

Mayo, A. (2001): The Human Value of the Enterprise, London.

Megna, P./Mueller, D.C. (1991): Profit Rates and Intangible Capital, in: The Review of Economics and Statistics, Jg. 73, S. 632-642.

Mueller, D.C. (1990): The Dynamics of Company Profits, Cambridge.

Mueller, D.C. (1986): Profits in the Long Run, Cambridge.

Nadiri, M.I./Prucha, I.R. (1996): Estimation of the Depreciation Rate of Physical and R\&D Capital in the U.S. Total Manufacturing Sector, in: Economic Inquiry, Jg. 34, Nr. 1, S. 43-56.

Nerlove, M./Arrow, K.J. (1962): Optimal Advertising Policy under Dynamic Conditions, in: Economica, Jg. 29, S. 129-142.

Odagiri, H. (1992): Growth through Competition, Competition through Growth- Strategic Management and the Economy in Japan, Oxford.

Odagiri, H./Haneda, S. (1998): Appropriation of returns from technological assets and the values of patents and R\&D in Japanese high-tech firms, in: Economics of Innovation and New Technology, Jg. 7, S. 303-321.

Odagiri, H./Yamawaki H. (1990): The persistence of profits in Japan, in: Mueller, D.C. (Hrsg.): The Dynamics of Company Profits, Cambridge, S. 129-146

Osterberg, W.P. (1989): Tobins q, Investment, and the Endogenous Adjustment of Financial Structure, in: Journal of Public Economics, Jg. 40, S. 293-318.

Perfect, S./Wiles, K. (1994): Alternative Constructions of Tobins q: An Empirical Comparison, in: Journal of Empirical Finance, Jg. 1, S. 313-341.

PhRMA (2006): Pharmaceutical Industry Profile 2006, Washington.

Porter, M.E. (1974): Consumer Behavior, Retailer Power and Market Performance in Consumer Goods Industries, in: The Review of Economics and Statistics, Jg. 56, S. 419-436.

PWC (2009): Bilanzierung von Ausgaben für Forschung und Entwicklung in der Pharma-Branche unter IAS/IFRS, Frankfurt.

Salamon, G.L. (1982): Cash recovery rates and measures of firm profitability, in: Accounting Review, Jg. 57, Nr. 2, S. 292-302.

Salmi, T. (1982): Estimating the internal rate of return from published financial statements, in: Journal of Business Finance and Accounting, Jg. 9, Nr. 1, S. 63-74.

Schmalensee, R. (1989): Inter-Industry Studies of Structure and Performance, in: Schmalensee, R./ Willig, R. (Hrsg.): Handbook of Industrial Organization, Amsterdam, North-Holland, S. 9511009.

Schäppi, H. (2002): Es ist an der Zeit, wieder radikal zu werden - Rentabilität und gesellschaftliche Bedürfnisse in der Medizin, in: Bulletin von Medicus Mundi Schweiz, Jg. 84, April.

Scherer, F.M. (2001): The link between gross profitability and pharmaceutical R\&D spending, in: Health Affairs, Jg. 20, Nr. 5, S. 216-220.

Schmalensee, R. (1972): The Economics of Advertising, Amsterdam.

Steward, T.A. (1997): Intellectual capital - the new wealth of organizations, New York.

Sveiby, K.E. (1997): The New Organizational Wealth: Managing and Measuring Knowledge-Based Assets, San Francisco.

Taylor, C. (1999): The cash recovery method of calculating profitability: an application to pharmaceutical firms, in: Review of Industrial Organization, Jg. 14, S. 135-146.

VFA [Verband Forschender Arzneimittelhersteller] (2009): Statistics 2009 - Die Arzneimittelindustrie in Deutschland, Berlin. 
Zambon, S./Lev, B./Abernethy, M./Wyatt, A./Bianchi, P./Labory, S. (2003): Study on the Measurement of Intangible Assets and Associated Reporting Practices, EU Kommission, Brüssel.

Jörg C. Mahlich, Dr., ist Univ.-Lektor am Institut für Volkswirtschaftslehre der Universität Wien.

Anschrift: Institut für Volkswirtschaftslehre, Universität Wien, Hohenstaufengasse 9, A-1010 Wien, Tel.: +43(0)1/4277-37404, Fax: +43(0)1/4277-9374, E-Mail: joerg.mahlich@univie.ac.at

B. Burcin Yurtoglu, Dr., ist Professor für Corporate Finance an der WHU - Otto Beisheim School of Management.

Anschrift: WHU - Otto Beisheim School of Management, Burgplatz 2, 56179 Vallendar, Tel.: +49 (0) 261/6509-710, Fax: +49 (0)261/6509-509, E-Mail: burcin.yurtoglu@whu.edu Für hilfreiche Kommentare danken wir Prof. Aloys Prinz (Universität Münster), Prof. Dennis Mueller (Universität Wien) und den anonymen Gutachtern. 